\title{
BMJ Open Impact of eye clinic liaison officers: a qualitative study in UK ophthalmology clinics
}

\author{
Mark Llewellyn, ${ }^{1}$ Jennifer Hilgart, ${ }^{1}$ Puja Joshi, ${ }^{2}$ Aelwyn Williams ${ }^{3}$
}

\begin{abstract}
To cite: Llewellyn M, Hilgart J, Joshi P, et al. Impact of eye clinic liaison officers: a qualitative study in UK ophthalmology clinics. BMJ Open 2019;9:e023385. doi:10.1136/ bmjopen-2018-023385

- Prepublication history for this paper is available online. To view these files, please visit the journal online (http://dx.doi. org/10.1136/bmjopen-2018023385).
\end{abstract}

Received 6 April 2018 Revised 4 December 2018 Accepted 22 January 2019

\section{Check for updates}

\section{(c) Author(s) (or their} employer(s)) 2019. Re-use permitted under CC BY-NC. No commercial re-use. See rights and permissions. Published by BMJ.

${ }^{1}$ Welsh Institute for Health and Social Care, University of South Wales, Pontypridd, UK

${ }^{2}$ Royal National Institute of Blind People, London, UK

${ }^{3}$ Swansea University, Swansea, UK

Correspondence to Professor Mark Llewellyn; Mark.Llewellyn@southwales. ac.uk

\begin{abstract}
Objectives To explore the impact of eye clinic liaison officers (ECLOs, also known as sight loss advisors) on the processes, functions and quality of ophthalmology clinics through the experiences of ophthalmology staff in the UK. Design Qualitative study.

Setting UK hospital ophthalmology clinics.

Participants Health and social care professionals in the UK.

Results ECLOs who had a presence in hospital ophthalmology clinics were seen as valuable in streamlining processes within the clinic, particularly in relation to the certification of visual impairment process, and providing continuity of care for patients when they were discharged from medical treatment. ECLOs also saved staff time in the clinic, as they were often responsible for providing emotional and practical support for patients living with sight loss.

Conclusions ECLOs are well placed in ophthalmology clinics. They can relieve pressure on clinical staff by taking on information giving and referring duties, allowing other staff to focus on their clinical responsibilities. The impact of ECLOs may depend on efficient communication with the clinical team, being trusted by other staff and having a good knowledge of local and national sight loss support services outside of the hospital setting. Further research could enhance our understanding of how much time and associated costs ECLOs substitute in the ophthalmology clinic.
\end{abstract}

\section{INTRODUCTION}

The impact of sight loss has been well documented, with significant effects on psychological well-being, social isolation, depression, mobility, being able to live independently, financial difficulties, employment and daily activities such as travel, shopping, cooking, reading, watching television and using technology. ${ }^{1-5}$ The need for information and support services for those living with sight loss has also received growing attention. There is considerable variation in the level of service provision across the UK. As noted by Gillespie-Gallery et al, financial concerns and a lack of cost estimates available in the literature constitute one the reasons why eye clinic liaison officer (ECLO) services have

\section{Strengths and limitations of this study}

This is the first study to qualitatively capture the impact of eye clinic liaison officers (ECLOs) in ophthalmology clinics by gathering the views of various health and social care professionals across the UK.

- The research design included clinics with varying levels of ECLO support, and although 30 sites across the UK were included in the study, the results may not be indicative of the impact of all ECLOs and other eye clinic support services.

- The potential for selection bias given the self-selecting nature of the participants is important to acknowledge.

- There were a large number of interviews conducted as part of the study, of varying length and depth. This was a function of trying to gather data from the 30 sites across the UK. On reflection, this approach may have precluded exploration of some issues in depth and did not really afford an opportunity for comparison between different models of ECLO service provision.

- Further research is needed to explore the most cost-effective model of ECLO support in terms of its impact on patients and the running of the clinic.

not been widely implemented. ${ }^{6}$ Following certification of visual impairment (CVI) or referral to social services, individuals may be offered a range of services including rehabilitation, mobility training, low vision aids, advice about welfare benefits and emotional support. Again, there is variation in the way these services are configured in the UK, with a variety of providers delivering rehabilitation using different strategies to operate between the health, social care and voluntary sectors. ${ }^{7}$ It can, therefore, be difficult for people to negotiate the complex networks of agencies involved in delivering these services-to ensure that they do not slip through the system when it comes to issues in respect of driving, for example-with people not knowing what is available and who to contact. ${ }^{5}$

There is also evidence that registration status, visual function and support needs 
change over time and that people should be given multiple opportunities to receive information and support for their visual impairment. ${ }^{8}$ People often access support and rehabilitation through clinical services and once they are discharged because nothing can be done for them clinically, they may not receive any information about other services that could benefit them. Therefore, access to services is often largely dependent on the initiative of the service user. ${ }^{8}$ This may be especially true for those who do not have a CVI, which is often the key to accessing vital financial, practical and social support.

Patients are able to access a range of clinical services in hospital eye clinics, including: medical diagnosis, treatment and advice by ophthalmic and optometric professionals. Patients might also receive non-medical support and advice. This type of service may be provided by, for example, nurses or volunteers from local charities. One study which interviewed health professionals from nine eye clinics in England found that services within the eye clinic differed from each other in terms of having clear paths of referral within the eye clinic, clear links with social services (or equivalent) and keeping detailed records. ${ }^{9}$ All the services provided a 'link service' referring patients onto others beyond the eye clinic. Since the publication of this study over 10 years ago, more formalised early intervention services have expanded in UK eye clinics, including the growth of the role of ECLOs, who may also be known as sight loss advisors or vision support officers, who can provide initial support and advice including emotional support and also signposting and referral to other sources of support. ECLOs can, therefore, be thought of as providing a link service for patients between healthcare, social care and the voluntary sector.

Other models of service delivery for eye clinic patients have also been developed, such as an emotional support and counselling service delivered within an integrated low vision service. ${ }^{1011}$ Previous research has demonstrated that ECLOs can help streamline the process of CVI and improve patient access to relevant support services. ${ }^{12}$ The current study was designed to evaluate the impact of ECLOs on patients' health-related quality of life, and its impact on the clinical team in the ophthalmology clinic. This paper focuses on the qualitative part of the study, wherein 141 interviews were conducted with clinical staff, ECLOs and other key informants across the UK. The interviews explored the staff perceptions of the impact of the ECLO on the clinic activity and patient pathways.

\section{METHODS}

Clinics were approached with the aim of recruiting clinics with a range of support services (eg, no formal support, ECLO provided by national or local charities, National Health Service (NHS) funded vision support officers) at a range of geographical locations (eg, large urban hospitals, rural district general hospitals). This stakeholder purposive sampling approach ${ }^{13}$ was determined as the most practical and expedient for the study to proceed, and in addition it ensured that a variety of contexts as described above had been considered in the study. While no specific inclusion or exclusion criteria were specified, it was important to ensure that all four health economies in the UK were included in the sample. Thirty sites in total were recruited. Two sites were recruited in Wales, three in Scotland, three in Northern Ireland and the remaining from England (including the Southwest, Southeast, Midlands, Northeast and Northwest). Twenty-six of these sites included ECLOs, which is approximately 3 in 10 of the total number of ECLOs operating $(n=91)$ in the UK (as of September 2016, according to the best estimate of the Royal National Institute of Blind People).

Contact was made with the clinical lead within each of the sites-typically this was the senior consultant ophthalmologist at each site. Once their permission had been obtained for a visit to occur, the study was registered with the relevant NHS Research and Development departments and a site visit date was arranged. Staff were informed of the site visit in advance, which usually lasted 1 day, and told they may be invited to take part in an interview. Potential participants were approached by the researchers on the day, and prior to the interview commencing, informed consent was obtained. Those staff content to speak to the researchers were interviewed in the clinic at a convenient time during the day, using private office space or empty clinics. Interviews were audio recorded with the consent of participants. There were a pragmatic set of inclusion criteria. Participants had to be employed within the clinic setting, or had to work in such a capacity that they would have a working knowledge of the role of ECLOs where such roles were present. That criteria satisfied, the approach was to ensure that as far as possible that there were a range of different participants recruited at each site (see table 1 ).

The interviews were conducted by two researchers $(\mathrm{JH}$ and ML) who are experienced in qualitative methods. The interviews intended to discover the impact of ECLOs

\begin{tabular}{lc}
\hline Table 1 Staff participants & $\begin{array}{l}\text { No of } \\
\text { interviews }\end{array}$ \\
\hline Participant & 26 \\
\hline Eye clinic liaison officer & 28 \\
\hline Nurse & 24 \\
\hline Consultant & 13 \\
Clinic/directorate manager & 12 \\
\hline Optometrist & 9 \\
\hline Commissioner (health and/or social services) & 8 \\
\hline Rehabilitation officer for visual impairment & 6 \\
\hline Orthoptist & 5 \\
Registrar/other doctor & 5 \\
\hline Medical secretary & 5 \\
\hline Others (including volunteers) & 141 \\
\hline Total & \\
\hline
\end{tabular}


on clinics by exploring how they operate within the clinic setting and what the impact is on clinic activity. For those clinics without any formal support services present, interviews explored how, where and by whom patients receive or get referred to further support services for their sight loss. One hundred and forty-one semistructured interviews with key staff such as ophthalmic consultants, ophthalmic nurses, administrative staff, ECLOs and rehabilitation officers for visual impairment (ROVIs) among others. Twenty-six of these interviews were completed over the telephone at a different time. Interviews ranged in length from 15 min to 2 hours, but usually lasted around half an hour. It is important to note that the quotations provided below come from different participants. Table 1 provides an account of who the researchers spoke to during the course of the study.

Interviews were transcribed verbatim and analysed with NVivo V.11 software, using a framework analysis approach $^{14}$ that is commonly used with such large qualitative datasets. This method takes into consideration preidentified issues that the researchers wish to investigate in accordance with the research questions, but allows flexibility for new themes to emerge. The framework approach was appropriate to explore the data as systematically as possible, given the multiple contexts and the sheer amount of data that was generated. It was especially useful here as it facilitated in-depth analyses of key themes across the whole data set, while simultaneously allowing for the views of each participant to remain connected to other aspects of their account. It also meant that comparing and contrasting data could be undertaken relatively easily which was crucial given the volume of interviews undertaken. Given its structure, the approach was useful as multiple researchers were working on the project, and was helpful in managing this large data set as maintaining a holistic overview of the entire data set was important. The subsequent construction of the thematic framework and other stages of analyses were agreed by all authors. Further information about how the conduct of the interviews met the consolidated criteria for reporting qualitative research is shown in the attached document.

\section{Patient and public involvement}

No patients or public were involved in this part of the study, but they were involved in other parts of the research project. In addition the views of patients, the public and stakeholders were sought on the design of the study.

\section{RESULTS}

\section{Streamlining CVI processes within the clinic}

There are numerous ECLO duties that seem to be common and every day within clinical and other services, and which ECLOs might be reasonably expected to perform. This theme encompasses how the activities ECLOs perform affect the capacity of each service: whether or not they add or subtract from that capacity in each clinic to work in the most efficient, patient-centred manner, and what are the effects ECLOs have on fundamental issues such as CVI registration, or the various pathways that have to be negotiated by patients. Efficiency in this context means the ability to improve the number of people seen in clinics, and their experience of those clinics, by providing an additional member of the team-the ECLO-to support the patient in the emotional, social and support (including providing non-clinical information and education) aspects of their care.

The participants suggested that many ECLOs had made CVI processes more efficient compared with previous systems that were in place. The ECLOs were often responsible for explaining the CVI process to the patient, filling out some of the paperwork with the patient (apart from the medical information completed by the consultant ophthalmologist) and answering any questions about the impact of the CVI on the future care of patients. The ECLOs, wherever they were based across the UK, would seem to be taking the bulk of the administrative burden.

'You often would find in the olden days piles of CVIs waiting in the consultant's office-that doesn't happen anymore' [Head of Optometry]

'Everything's now filled out properly and there's proper processes in place for everything and that is really important, especially for the doctors. That's because they can get on and see patients quicker, so they can register the patient and then any patient that wants to ask they can go straight to [the ECLO] and she can take them away from the doctor and the doctor can get on with his patients' [Senior Nurse]

Furthermore, many of the participants reiterated that ECLOs seem to have 'sorted out' the CVI pathway in a comprehensive manner. Repeated accounts were given of how the CVI process had previously been severely blocked in many places. Participants reported inefficiencies such as people 'slipping through the system', for example, driving when they should not be driving or not being assessed at home. With an ECLO in place, many of these problems simply do not exist anymore. ECLOs in clinics often facilitate the smooth running of the CVI pathway in practical ways, right from the start, by making sure the doctors have the right forms or by flagging up certification requests which have not happened. In several sites with ECLO support, the majority of CVIs were now being processed in line with national guidance.

I'll go through the [CVI] and make sure it's all complete, then I'll sort out getting it duplicated. I will put the copy in the hospital records, I will send a copy to the GP, the patient, the local vision support services and a copy for [the hospital]. I go right through it because we were finding CVIs in patients' notes 2 years later. My whole ethic is I want to stick to the Department of Health guidelines that when a CVI is completed it gets to social services within 5 working days and over $90 \%$ of them do. [ECLO] 
In several areas, it was suggested that without ECLO support this process would not be completed in a timely way, which apart from the risks highlighted above, also had implications in terms of getting the appropriate support which can make a real difference in the lives of people.

What used to happen here was they were sent to the local CCG or primary care trust at that point...then the trust would send them in batches to social services, so it was at least 6 months before they were seeing the patient [ECLO]

In one site where the medical secretaries used to process the CVIs, it was acknowledged that they did not have the resource to ensure the CVI was processed immediately or that it was being followed up by social services.

It's great at the moment because we're behind in our typing, that form might sit there for a week, two weeks before it gets processed. Whereas now if we can see there's one sitting there, we can give it straight to [ECLO] and he can process it which is obviously going to speed everything up and help the patient. [(Medical secretary]

\section{Adding capacity}

Many participants were convinced that having an ECLO in post saves a lot of clinical and administrative time, whether clinical managers, matrons, nurses, consultants and others. Generally speaking, participants acknowledged that they save time primarily by taking pressure off the medical staff, who themselves are often under pressure to move people through the system quite quickly, more so with ever-tightening budgets. As one participant pointed out 'it releases some of the time for the trained staff to do what they trained for'.

Overwhelmingly, most participants thought that ECLOs contribute to the smooth running of eye clinics. Many of the clinical staff reported that they simply do not have the time to provide emotional support and practical advice to patients about living with sight loss. Having the ECLO on hand to refer patients who required extra support about local support groups or information about welfare benefits, for example, meant that the clinical staff could focus on their clinical duties.

I would suggest to people that ECLOs have an impact on the throughput of a clinic and allow us to see as many people as possible, and also that given the very positive feedback we've had about their support, that there is a direct service quality and patient benefit argument for having one in any clinic [Consultant Ophthalmologist]

I think the ECLOs have a fantastic role in that they take away the patient from the clinical side... where the treatment has been given or terminated or could not be completed for one reason or other...and they get them prepared for life after. So from being sympathetic as to what their clinical condition is they quickly move onto what is available and accessible outside... and also what is available here in the hospital... [Consultant Ophthalmologist]

Interestingly, clinical staff did not identify any negative impacts of this, such as whether having the additional capacity of an ECLO would serve to 'de-skill' them in respect of the emotional, social and support needs of patients. Instead, while they recognised that they still had such responsibilities in respect of these issues for their patients and would spend an amount of time undertaking these tasks, they found that the ability to use the skills and time of ECLOs enhanced and added capacity to the 'offer' within the clinic.

ECLOs would seem to enhance efficiency within clinics and streamline processes to release capacity, not only through the CVI process as outlined, but by referring and signposting appropriately and according to the needs of patients, in a timely way; '... (referring to an ECLO) it's not the case of passing the buck, it's about putting the patient in the right place for the right reasons at the right time-that's how it works' (Orthoptist). The additional capacity provided by the ECLO meant that the clinical staff were able to concentrate on their clinical duties, and this was seen to make for a better quality service for patients. 'I think that it's a poor service without an ECLO, having someone on tap makes a big difference' (Consultant Ophthalmologist).

In sites where no formal support services were available, the supportive duties were often done by volunteers or by nursing staff. Administrative duties involved in the processing of a CVI were often the responsibility of consultant secretaries. When reflecting on their roles before the ECLO came into post, staff from one site reported that it was often difficult to fit in the 'ECLO-type' work-developing and maintaining knowledge about local groups and networks, and ensuring that people are appropriately signposted to them-around their other duties. Some staff also found it difficult to provide patients with information as they did not have sufficient knowledge of what is available for patients.

I used to be the nurse that registers patients, I was kind of the ECLO nurse before [name of ECLO] came, and it's such a relief that [name of ECLO is] here and it's such a great service he's providing because we used to have our nursing duties and I had clinics ... From my point of view I did my absolute best but it's such a relief because sometimes I'd spend an hour and come out emotionally drained from it all and have to crack on with my nurses duties. And sometimes I couldn't take that time out and I'd have to phone them at home or ask them to come back in ... the whole thing was very unsatisfactory. [Ophthalmic nurse]

If the patient rings up and asks a direct question, we have to try and find the information for them. I have 
spent hours on the phone trying to get through to various organisations, social services, to try and get the answer to the question without much success-so we pass them all to [the ECLO] now. He does save us time and he knows what he's talking about, we don't. Like what benefits they are entitled to and if they can get a blue badge. [Medical secretary]

In other areas that operated without an ECLO, staff at times stated that they struggle to keep up with the emotional and support requirements of patients, and being unable to offer the kind of specialised advice for example, around benefits, which ECLOs often give. Any advice given was 'ad hoc'. For many sites operating without an ECLO, it was suggested that having an ECLO would vastly improve their service. The majority of those that were interviewed in sites without ECLOs were very keen to ensure that they had such a post as part of their team as soon as it was possible for this to happen as they could identify the benefits of such posts. Some were in the process of recruiting, and clinical staff were looking forward to having one as part of their team.

Yes certainly being able to reduce time to be able to say 'look I can now hand you over to my colleague who'll be able to discuss more of the implications of this and what the benefits might be. [Consultant Ophthalmologist]

\section{Maintaining engagement and continuity of care}

Clinical staff reported that when people find it difficult to accept their diagnosis, often the day-to-day reality of running a clinic is that there is no more clinical time to help them, so it is often up to the ECLO to step in. By doing this, many of the participants believed that the presence of ECLOs in clinical eye services makes them more of a 'complete' service, in contrast to pre-ECLO times where there was less support, and the support was less structured when offered.

We used to say 'sorry there is nothing we can do for you'-now we can send them to [the ECLO] for support. We have a moral duty to give patients the best possible treatment and it's nice to give them something positive in the form of ongoing support from the ECLO' [Consultant Ophthalmologist]

'When we refer them to (the ECLO), it means we haven't finished with the patient and it means that we don't have the expertise...' [Consultant Ophthalmologist]

The ECLOs were trusted as having the necessary skills and knowledge to support patients in the long term. ECLOs also provided a point of contact for patients if they had any further support needs related to their visual impairment, which was seen as making a big improvement to the continuity of care the eye clinic provides.

'ECLOs mean that you feel that the patient is saferyou pass over to a person who is more specialised to follow up, and it also saves time' [Consultant Ophthalmologist]

'You've not left the patient to go home wondering if they're safe, .... whereas the doctors and nurses they don't have the time and they don't have the skills just for that purpose because there are so many other things clinically to do. If I have a patient, I do encourage them to speak with [the ECLO]. It makes a difference and you feel safer' [Clinic manager]

Many other participants indicated that it is often the ECLO who does a lot of the follow-up work to make sure that care continues beyond the medical treatment of the clinic. By supporting the patient throughout their journey, participants reported that it helps other professionals in community support services to maintain their engagement. For example, joint working with ROVIs is far easier when the ECLO is involved with the patient, so when looking at a more integrated model of health and social services for visually impaired citizens, ECLOs are key to delivering some of this. By taking more time to explain the process, participants noted how this reduces the stigma felt by some, especially older patients, if there is a referral to social services; '... when then I go and actually make contact by phone, and then do the home visit to them, generally I'm finding the person is a lot more accepting' (ROVI). Crucially, ECLOs were reported to be more likely to follow up on the process, including setting up any further support from that point onwards, in effect helping to 'triage' people appropriately for example, with social services or others; 'The ECLO can help me to weigh up if it's safe to put them on the waiting list or actually we should get people out here much more quickly' (Sensory Team Manager, Social Services). People from within and outside the clinical team stated that they turn to the ECLO for help when they have any non-medical patient issues; in many localities they are the trusted first port of call.

Equally important, because of the bidirectional nature of the ECLO within the system-acting as a bridge between information coming from the clinical team to the patient, and ensuring that the voice of the patient is heard by the clinical team-engagement with the world outside of the clinic is made available to the rest of the clinical team: many ECLOs were proactive in providing updates on subjects such as changes to benefits, changes to the local voluntary sector provision or any other services available outside of the clinic. This can then translate into practical help being offered by the whole team, not just the ECLO.

'[The ECLO] gives them information about what financial support, what other services are available, what other support is out in the community as well, it's not just in-house, she has a whole remit out there where she can give patient connections...It's a holistic approach really because not only does she give the visual aids and the internal support....she also helps me with other things in clinics, gaining 
information for other specialties and things like that' [Matron]

\section{DISCUSSION}

From the research, we would suggest that ECLOs have a beneficial and welcome impact on the activities of most clinics. They are often involved in key processes and 'pathways' which have a large impact on helping patients to progress and receive the care needed in the various systems that they have to negotiate. From the accounts given, ECLOs can help to speed up and take the pressure off certain aspects of clinic activity, thereby freeing clinicians to see more patients. For example, many are involved in the CVI registration process; what was seemingly quite an ad hoc system in many places has been largely improved. However, there are only a small number of settings in which ECLOs have been integrated into the formal NHS care pathway (there were two of these in our sample of site visits) - which explains the use of inverted commas when 'pathways' was discussed above. The implication of this is to corroborate the findings of previous studies which have suggested that gaps in signposting and referral may persist (depending on the role of the individual ECLO) unless the ECLO is formally incorporated as part of the care pathway. ${ }^{5}$

The strength of this study lies in the number and range of staff that were interviewed. That said, many of the interviews were fairly brief due to the busy schedule of clinical staff. Although 30 ophthalmology clinics across the UK were included in the study, the results may be generalisable to other ECLOs and eye clinic support services. It is difficult to be precise, but we did not experience significant variation in the type of data gathered across the sites, which leads us to conclude that there is unlikely to be significant further variation in findings across other clinics in the UK.

Much of the impact of the ECLOs were dependant on local context such as support of the ECLO post from senior clinical and managerial staff and the perception of the ECLO as being trustworthy and knowledgeable. Further, it is important to acknowledge that limitations like selection bias have the potential to operate is such studies, and concerns over the difficulties for participants to express negative views about close colleagues are valid. That said no such negative views about ECLOs were expressed, even when participants were asked about such matters.

The study raises the question of whether there is a sufficient benefit of ECLOs to recommend that all ophthalmology clinics would benefit from having an ECLO based in the clinic. They certainly appear to be of benefit to patients (from the perspective of clinical staff) and also make efficiencies within the clinic. Of course, this depends on the skills and motivation of the individual ECLO, but we did not find any who were viewed in a negative light by clinical staff. Some staff did not have much communication with the ECLO due to their particular role within the clinic, but all were aware of the ECLOs roles and responsibilities. One implication of additional capacity being provided by ECLOs is the potential for other clinical roles to become more specialised, focusing less on the whole person (as an ECLO would) and more on their eye condition.

The current study findings are in line with that of previous research of the CVI process, whereby ECLOs were found to streamline the CVI process, making it more efficient and thereby granting patients more timely access to support services. ${ }^{12}$ The current study extends those findings by demonstrating that by streamlining these processes and by bridging the gap between health and social or third sector support services, the ECLOs can save clinical staff time, allowing them to focus on their clinical duties. The benefits for patients were also well highlighted by participants, who were confident that the ECLOs provided crucial emotional support and were a point of contact for patients who needed further support in the future.

It is difficult to be certain about the optimum level of ECLO support needed in clinics which would lead to the greatest benefit to the clinic and to patients. For example, do all clinics need a full-time ECLO based in the clinic? Does it make a difference to the impact if the ECLO is employed by the NHS or a third sector organisation? Further research could quantitatively unpick these issues. What is clear from previous research is that patients with sight loss can have significant information and support needs,${ }^{57}$ and the current study posits that ECLOs are well suited to meet those needs.

Acknowledgements The authors wish to thank all individuals who took part in an interview and to the RNIB for their comments on the article.

Contributors $\mathrm{JH}, \mathrm{ML}, \mathrm{AW}$ and $\mathrm{PJ}$ analysed the data. $\mathrm{JH}$ wrote the initial draft and all authors revised the initial draft. ML and $\mathrm{JH}$ wrote the final draft. ML made the revisions following feedback from the reviewers.

Funding The Royal National Institute of Blind People (RNIB) funded this research. The funders contributed to the design of the research. The RNIB is one (among a number of other) funders of sight loss advisor and ECLO posts across the UK.

Competing interests None declared.

Patient consent for publication Not required.

Ethics approval Ethical approval for the study was obtained from the Faculty of Life Sciences and Education Ethics Committee of the University of South Wales prior to the research being conducted.

Provenance and peer review Not commissioned; externally peer reviewed. Data sharing statement None of the unpublished data from the study is available.

Open access This is an open access article distributed in accordance with the Creative Commons Attribution Non Commercial (CC BY-NC 4.0) license, which permits others to distribute, remix, adapt, build upon this work non-commercially, and license their derivative works on different terms, provided the original work is properly cited, appropriate credit is given, any changes made indicated, and the use is non-commercial. See: http://creativecommons.org/licenses/by-nc/4.0/.

\section{REFERENCES}

1. Hodge S, Loneliness EF. social isolation and sight loss: a literature review conducted for Thomas Pocklington Trust. Lancaster: Lancaster University, 2013.

2. Cimarolli VR, Boerner K, Brennan-Ing M, et al. Challenges faced by older adults with vision loss: a qualitative study with implications for rehabilitation. Clin Rehabil 2012;26:748-57. 
3. Rees G, Tee HW, Marella M, et al. Vision-specific distress and depressive symptoms in people with vision impairment. Invest Ophthalmol Vis Sci 2010;51:2891-6.

4. Social S, Research M. Understanding the needs of blind and partially sighted people: Their experiences, perspectives and expectations: University of Surrey, 2009.

5. Thetford C, Robinson J, Knox P, et al. Long-term access to support for people with sight loss. Br J Vis Impair 2011;29:46-59.

6. Gillespie-Gallery H, Subramanian A, Conway ML. Micro-costing the provision of emotional support and information in UK eye clinics. BMC Health Serv Res 2013;13:482.

7. Dickinson C, Linck P, Tudor-Edwards R, et al. A profile of low vision services in England: the Low Vision Service Model Evaluation (LOVSME) project. Eye 2011;25:829-31.

8. Hodge S, Thetford C, Knox P, et al. Finding your own way around: experiences of health and social care provision for people with a visual impairment in the United Kingdom. Br J Vis Impair 2015;33:200-11.
9. Douglas G, Spurgeon P, Pavey S. An exploratory study of the impact of non-medical eye clinic support services (ECSS) in hospital eye clinics. Int Congr Ser 2005;1282:105-8.

10. Hodge S, Barr W, Bowen L, et al. Exploring the role of an emotional support and counselling service for people with visual impairments. Br J Vis Impair 2013;31:5-19.

11. Barr W, Hodge S, Leeven M, et al. Emotional support and counselling for people with visual impairment: Quantitative findings from a mixed methods pilot study. Couns Psychother Res 2012;12:294-302.

12. Boyce T, Leamon S, Slade J, et al. Certification for vision impairment: researching perceptions, processes and practicalities in health and social care professionals and patients. BMJ Open 2014;4:e004319.

13. Palys T. The sage encyclopedia of qualitative research methods. Given LM, ed. Purposive sampling. Los Angeles: Sage, 2008:697.

14. Ritchie J, Spencer L. Qualitative data analysis for applied policy research. In: Burgess R, Bryman A, eds. Analyzing qualitative data. London: Sage, 1994:173-94. 